\title{
Object-Oriented Modeling and Simulation of the Arterial Pressure Control System by Using MODELICA
}

\author{
J. Fernandez de Canete ${ }^{1}$, J. Luque ${ }^{2}$, J. Barbancho², and V. Muñoz ${ }^{1}$ \\ ${ }^{1}$ Dpt. System Engineering and Automation, Andalucia-Tech University, Malaga, Spain \\ ${ }^{2}$ Dpt. Electronics, Andalucia-Tech University, Sevilla, Spain
}

\begin{abstract}
The modeling of physiological control systems via mathematical equations reflects the calculation procedure more than the structure of the real system modeled, and several simulation environment have been used so far for this task. Nevertheless, object-oriented modeling is spreading in current simulation environments through the use of the individual components of the model and its interconnections to define the underlying dynamic equations. In this paper we describe the use of the MODELICA $A_{T M}$ simulation environment in the object-oriented modeling of the cardiovascular control system. The performance of the controlled system has been analyzed by object-oriented simulation in the light of existing hypothesis and physiological data used here for validation purposes.
\end{abstract}

Keywords-Object-Oriented Modeling, MODELICA Simulation Language, Cardiovascular System, Pressure Control.

\section{INTRODUCTION}

There are a considerable number of specialized and general-purpose modeling software applications available for biomedical studies, which are commonly divided into structure-oriented and equation-oriented. Most of them follow a causal modeling approach and require explicit coding of mathematical model equations or representation of systems in a graphical notation such as block diagrams, which is quite different from common representation of physiological knowledge.

The object-oriented approach can offer many advantages in biomedical research both for the building of complex multidisciplinary models when dynamics are given by a set of differential algebraic equations [1]. The object-oriented approach has been made possible by using the modeling environment MODELICA ${ }_{\mathrm{TM}}$ [2] among others, which allows the system, subsystem, or component levels of a whole physiological system to be described in increasing detail. While the MODELICA $\mathrm{TM}_{\mathrm{TM}}$ environment has been used for a long time in different fields of engineering [3] there are few results in biomedical system modeling [4-5] particularly in cardiovascular modeling and control.

Cardiovascular modeling and control present a particular challenge and require both a multi-scale and a multi-physics approach [6-7]. Several mathematical models of the closed loop cardiovascular system have been developed [8-9] and software tools based on hierarchical block diagrams have also been applied to the description of cardiovascular systems [10-11].

The regulatory control of the cardiovascular system has been studied intensively, more than any other physiological system. In fact, the high rate of cardiovascular diseases is certainly one of the main reasons for its analysis. The control mechanisms responsible for maintaining arterial blood pressure may be divided into short-term processes, which are effective over a period of seconds to hours, and longterm processes that operate over days to weeks. The former are largely neural based, utilizing receptors in the heart and blood vessels to sense blood pressure and the autonomic nervous system (ANS) to regulate the cardiac function and diameter of resistance vessels. The mid-term control is basically hormonal while the renal system plays the central role at long-term [6].

In this paper we describe the use of the MODELICA ${ }_{T M}$ simulation environment in the object-oriented modeling of the cardiovascular regulatory system considering the short, medium and long-term mechanisms. For this task we have followed an acausal hierarchical structure whose validity has been previously assessed to represent the cardiovascular dynamics as a multi-compartmental system. The results have been obtained under both physiological and pathological conditions, namely, hypertension due to either increased values of peripheral resistance, heart rate or intravascular volume, and hypotension due to either decreased heart rate or severe hemorrhage, assuming the validation tests previously performed with physiological data.

\section{MODELING THE CARDIOVASCULAR CONTROL SYSTEM}

The cardiovascular model here used can be represented with a set of nonlinear equations describing its dynamics [8]. Nevertheless, the present paper focuses on the multicompartmental acausal approach so that the whole system will be described by eight compartments, namely, the four cardiac chambers (auricles and ventricles), the pulmonary circulation (arterial and venous) and the systemic circulation (arterial and venous) (Fig. 1). 


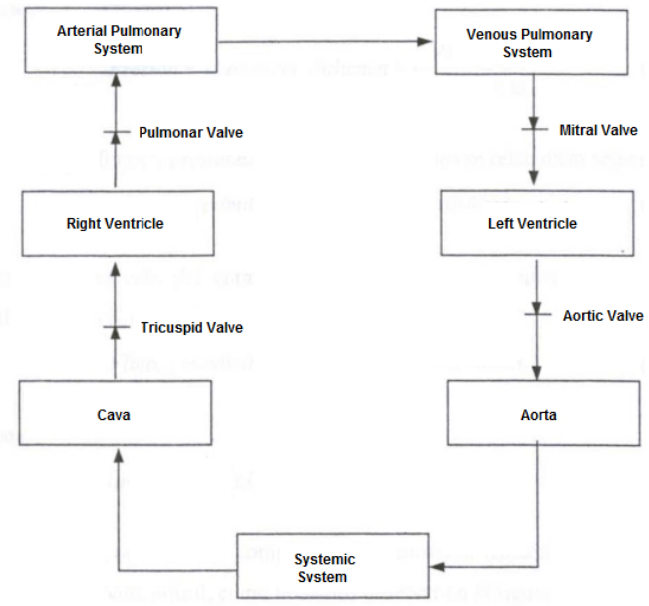

Fig. 1 Compartmental Model of the Circulatory System

Each compartment will be modeled by using a mathematical relationship between pressure $P_{i}(t)$, volume $V_{i}(t)$, input flowrate $F_{i_{i n}}(t)$ and output flowrate $F_{i_{o u t}}(t)$ relative to the $i^{\text {th }}$ compartment given as

$$
\begin{gathered}
\frac{d V_{i}(t)}{d t}=F_{i_{\text {in }}}(t)-F_{i_{\text {out }}}(t) \\
P_{i}(t)=\frac{V_{i}(t)}{C_{i}}
\end{gathered}
$$

while flowrate $F_{i j}(t)$ between compartments $i$ and $j$ is defined by

$$
\begin{gathered}
F_{i j}(t)=E_{i}\left(P_{i}(t)-P_{j}(t)\right) \\
F_{i j}(t)=E_{i} \lim \left(P_{i}(t)-P_{j}(t)\right)
\end{gathered}
$$

where $C_{i}$ and $E_{i}$ stand respectively for the compliance and admittance of compartment $i$, while the function $\lim (x)=$ $\max (x, 0)$ is used in case of flowrate through a valve. It is important to highlight the temporal dependence $E_{i}=E_{i}(t)$ which exhibits each of the ventricular compartments.

The short-term mechanism to control arterial pressure is represented by the baroreceptors, which as described in [12] regulate the heart rate, the ventricular elastance, the cava elastance and the peripheral system resistance as a function of the mean aortic pressure value through a first-order filter with transfer function $H_{j}(s)$ and a dead zone (Fig. 2).

The long-term mechanism to control arterial pressure is represented by the renal system through the elimination of urine. In order to model this effect it is assumed as described in [13] that there is a linear relation between the renal excretion flowrate $J_{u}(t)$ and the plasmatic volume $V_{p}(t)$ stated as

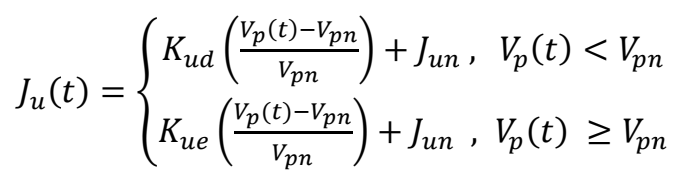

where $K_{u d}, K_{u e}$ and $J_{u n}$ represent a measured excretion constant for dehydration and overhydration and the urine flow under normal condition respectively while $V_{p n}$ stands for the plasmatic volume under physiologic conditions. The plasmatic volume $V_{p}(t)$ is calculated by aggregation of the volume $V_{i}(t)$ of each of the constitutive compartments applying the hematocrit factor.

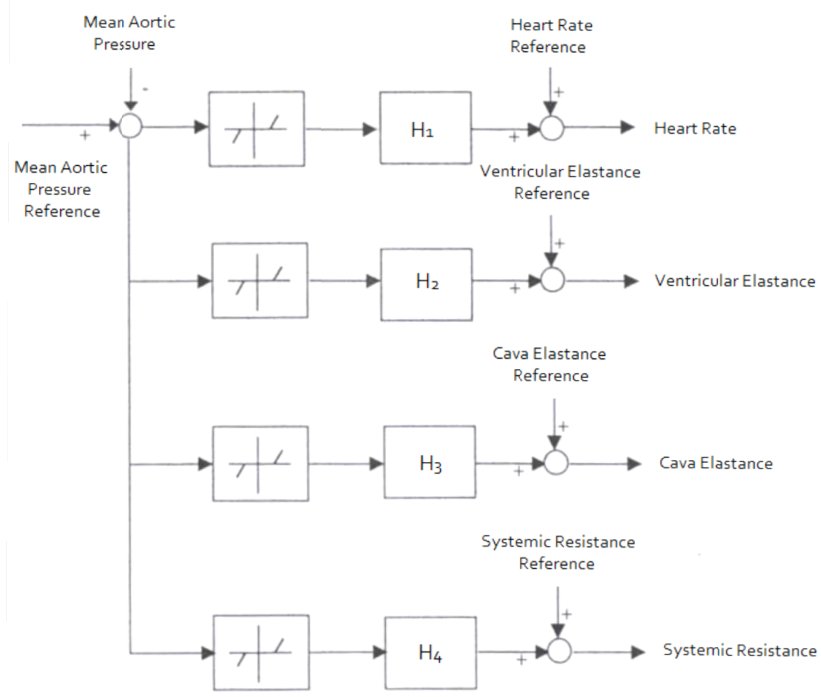

Fig. 2 Diagram of the Baroreceptor Regulatory System

The whole cardiovascular control system is obtained by integration of the circulatory system, the short-term and the long-term arterial pressure control modules described.

\section{THE MODELICA ${ }_{\mathrm{TM}}$ MODELING APPROACH}

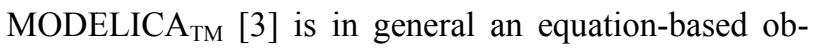
ject-oriented language for modeling continuous complex physical systems for the purpose of computer simulation, and is primarily based on equations instead of assignment statements. It also has multi-domain modeling capability, meaning that model components corresponding to physical objects from several different domains can be described 
and connected. MODELICA $\mathrm{TM}_{\mathrm{TM}}$ is an object-oriented language whose basic construct is class (model, block, function, type,...), which facilitates reuse of components and evolution of models.

The most important difference with regard to the traditional block-oriented simulation tools is in the different way of connecting components. So, a special-purpose class connector as an interface defines the variables of the model shared with other sub-models, without prejudicing any kind of computational order. In this way the connections can be, besides inheritance concepts, thought of as one of the key features of oriented-object modeling, enabling effective model reuse.

The basic idea of implementation in MODELICA $\mathrm{TM}_{\mathrm{TM}}$ is to decompose the described system into components that are as simple as possible and then to start from the bottom up, connecting basic components (classes) into more complicated classes, until the top-level model is achieved. The default integration method is the DASSL code as defined by [14], nevertheless some other methods are available as Runge-Kutta and BDF based.

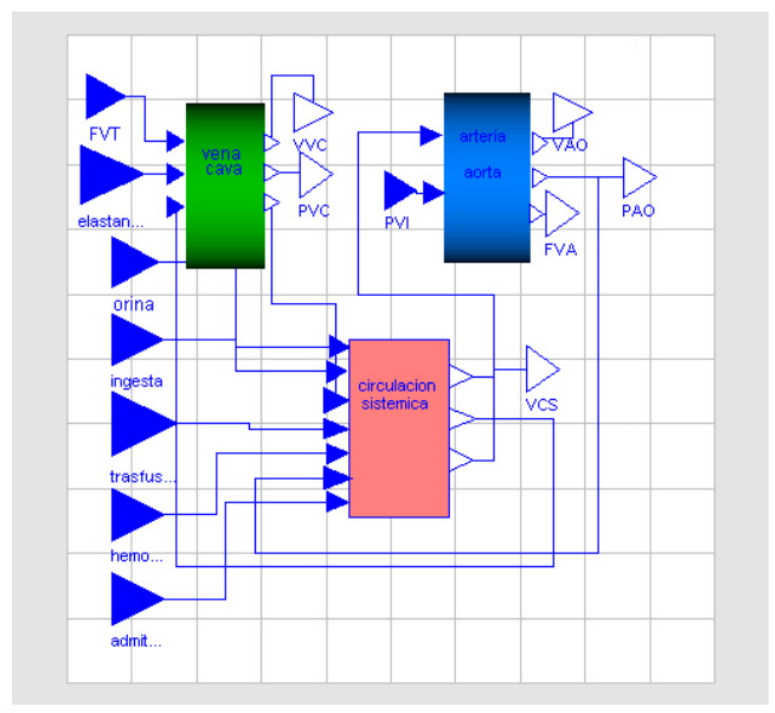

Fig. 3 MODELICA $_{\mathrm{TM}}$ Block Diagram of the Systemic Circulation

The arterial pressure control system was simulated using MODELICA $_{\mathrm{TM}}$ including each of the circulatory compartments above mentioned together with the control mechanisms. The MODELICA TM $_{\mathrm{TM}}$ block diagrams of any of the compartments are illustrated in Fig. 3, while the baroreceptor control system and the renal control system programming are also depicted in Fig. 4 and Fig. 5.

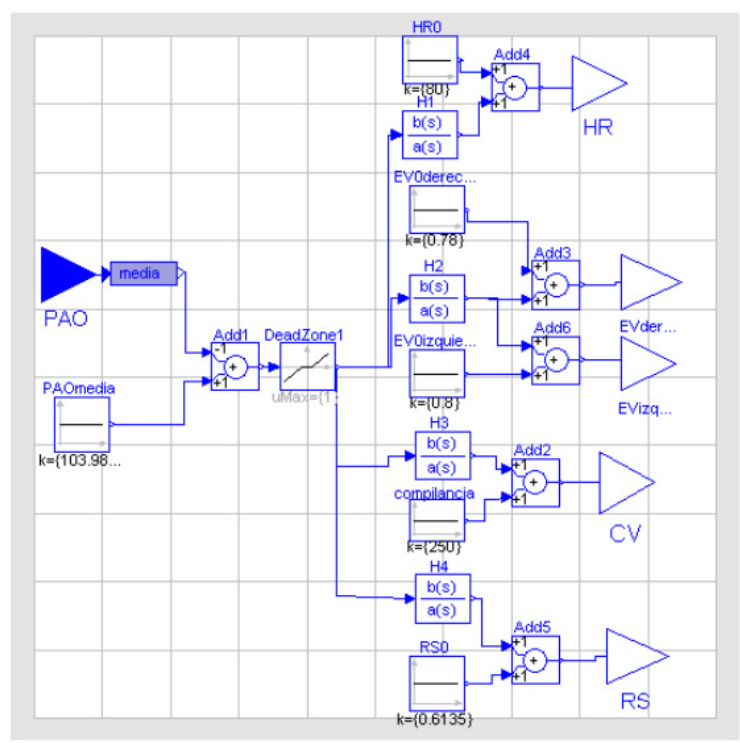

Fig. 4 MODELICA $_{\mathrm{TM}}$ Block Diagram of the Baroreceptor Mechanism

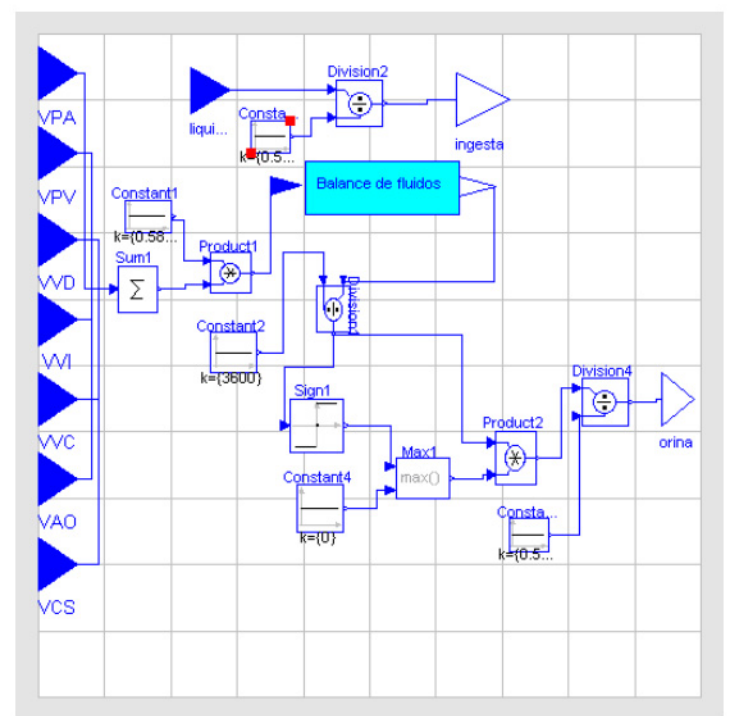

Fig. 5 MODELICA $_{\text {TM }}$ Block Diagram of the Renal Control Mechanism

\section{RESULTS}

In order to test the performance of the pressure control system several experiences have been accomplished, both under physiologic conditions by varying resistance,elastance or heart rate reference values in the baroreceptor control module and by considering liquid intake, hemorrhage and blood transfusion as external flowrates entering the circulatory system. The results have been 
obtained by assuming the validation tests previously performed with physiological data by [8].

In Fig. 6 it is depicted the short-term control system response to a system resistance increasing showing the mean aortic pressure regulation to a constant reference level.

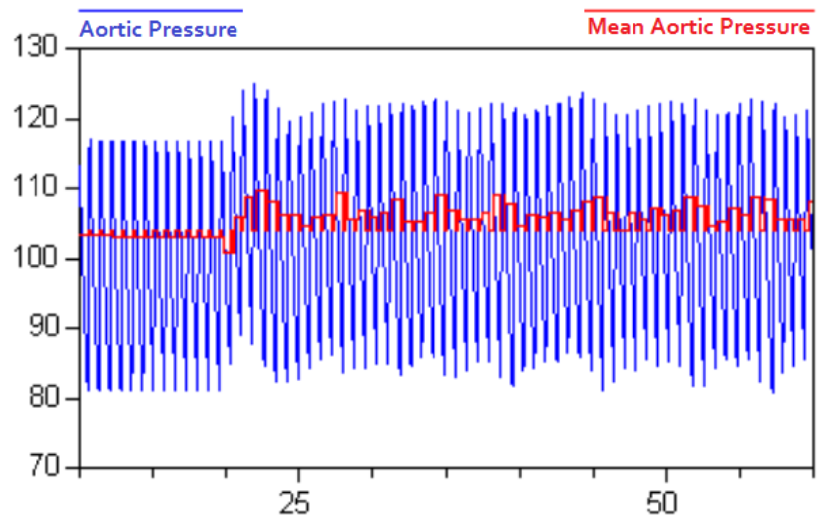

Fig. 6 Baroreceptor response to a sudden $30 \%$ change in the system resistance at $\mathrm{t}=20 \mathrm{~s}$

In Fig. 7 it is shown the long-term control response to a slight hemorrhage combined with a transfusion applied to the system circulation compartment. It can be observed the systemic pressure value is restored after the transient showing the ability of the renal system to compensate for these abnormal situations.

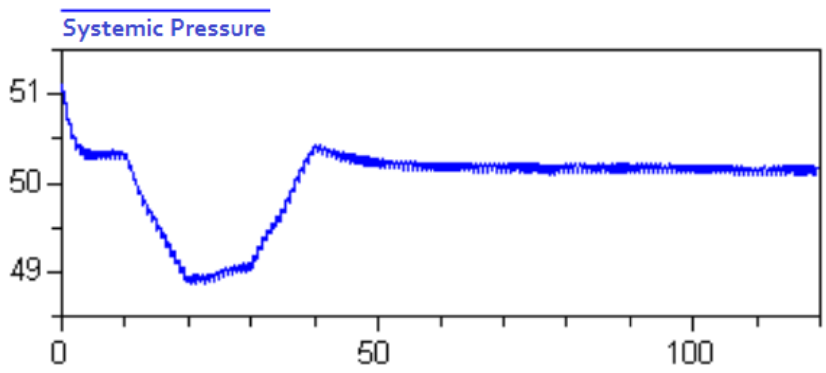

Fig. 7 Renal response to an hemorrhage and subsequent transfusion of 10 $\mathrm{ml} / \mathrm{min}$ during $10 \mathrm{~min}$ at $\mathrm{t}=10 \mathrm{~s}$ and $\mathrm{t}=30 \mathrm{~s}$ respectively

\section{CONCLUSIONS}

An object oriented computer model of the short- and long- term arterial pressure control system under both physiological and pathological condition is presented.
The whole system has been modeled and simulated under the MODELICA $\mathrm{TM}_{\mathrm{TM}}$ integrated environment, which uses a hierarchical modeling strategy which aids to a quantitative understanding of the control process.

The described approach represents also a valuable tool in the teaching of physiology for graduate medical students.

Future works consider the application of the proposed approach to the optimized performance of a patient undergoing hemodialysis treatment. Also, the development of a MODELICA $_{\mathrm{TM}}$ Physiological Library for the building of cardiovascular and respiratory models is currently under construction.

\section{REFERENCES}

1. Hakman M, Groth T. (1999). Object-oriented biomedical system modelling:/The rationale. Comput. Methods Programs Biomed. 59:117.

2. Mattsson SE., Elmquist H, Otter M (1998). Physical system modelling with Modelica,. Control Eng. Practice 6:501-510.

3. Tiller M. (2001). Introduction to physical modeling with MODELICA, Kluwer Ac. Press.

4. Cellier FE., Nebot A. Object-oriented modeling in the service of medicine, Proc. $6^{\text {th }}$ Asia Simulation Conference, Beijing, China, 2005, pp 33-40.

5. Fernandez de Canete J, Del Saz Huang P. (2010). First-principles modeling of fluid and solute exchange in the human during normal and hemodialysis conditions. Comput. Biol. Med. 40:740-750.

6. Guyton AC, Hall JE (1996). Textbook of medical physiology, 9th Edition, W.B. Saunders, New York, NY.

7. Berne RM, Levy MN (1997). Cardiovascular physiology, Mosby, St. Louis.

8. Avanzolini G, Barbini P, Cappello A, Cevenini G (1988). CACDS simulation of the closed-loop cardiovascular system. Int. J. Biomed. Comput. 22:39-49.

9. Rothe RF, Gersting JM (2002). Cardiovascular interactions: an interactive tutorial and mathematical model, Am. J. Physiol. Adv. Physiol. Educ. $26: 98-109$.

10. Raymond GM, Butterworth E, Bassingthwaighte JB (2003). JSIM: Free software package for teaching physiological modeling and research. Exp. Biol. 280:102-107.

11. Abram SR, Hodnett BL, Summers RL, Coleman TG, Hester RL (2007). Quantitative circulatory physiology. An integrative mathematical model of human mathematical model of human physiology for medical education, Adv. Physiol. Educ. 31:202-210.

12. Kline I (1988). Handbook of biomedical engineering. Academic Press, London.

13. Gyenge C, Bowen BD, Reed RK, Bert JL (2003). Mathematical Model of Renal Elimination of Fluid and Small Ions during Hyper and Hypovolemic conditions. Acta Anaest. Scand. 47:127-137.

14. Brenan KE, Campbell SL, Petzold LR (2011). Numerical solution of initial value problems in differential algebraic equations, SIAM, 2nd edition. 